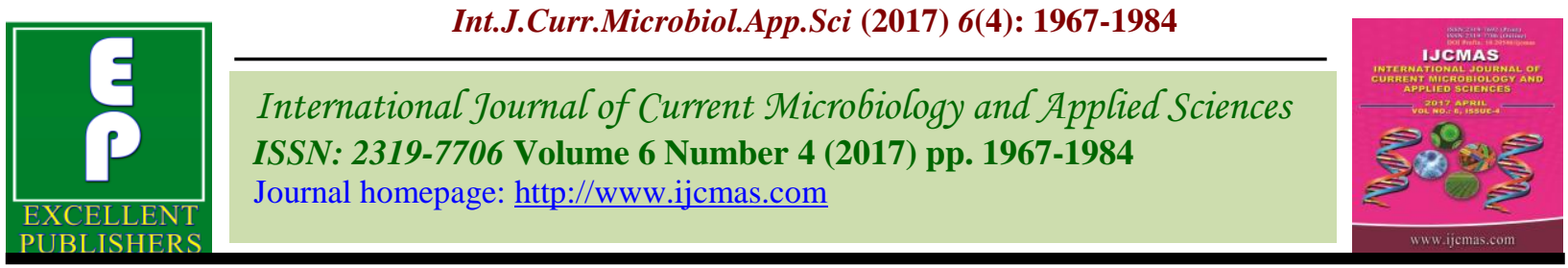

Review Article

https://doi.org/10.20546/ijcmas.2017.604.235

\title{
Recent Advances in QTL Mapping and Quantitative Disease Resistance Approach
}

\author{
Fayaz Ahmad Sheikh* \\ Division of Genetics and Plant Breeding, Sher-e-Kashmir University of \\ Agricultural Sciences and Technology, Kashmir, India \\ *Corresponding author
}

\section{A B S T R A C T}

\section{Keywords}

QTL mapping,

Quantitative

Disease Resistance (QDR),

Nectar, Markers, rice blast fungus.

\section{Article Info}

\section{Accepted:}

15 March 2017

Available Online:

10 April 2017
Quantitative trait locus (QTL) mapping is a highly effective approach for studying genetically complex forms of plant disease resistance. With QTL mapping, the roles of specific resistance loci can be described, race-specificity of partial resistance genes can be assessed and interactions between resistance genes, plant development and the environment can be analysed (Young, 1996). DNA markers tightly linked to quantitative resistance loci (QRLs) controlling QDR can be used for marker-assisted selection (MAS) to incorporate these valuable traits. QDR confers a reduction, rather than lack, of disease and has diverse biological and molecular bases as revealed by cloning of QRLs and identification of the candidate gene(s) underlying QRLs (Dina St. Clair, 2010). Outstanding examples include: quantitative resistance to the rice blast fungus, late blight of potato, bacterial wilt of tomato, and the soybean cyst nematode. These studies provide insights into the number of quantitative resistance loci involved in complex disease resistance, epistasis and environmental interactions, race-specificity of partial resistance loci, interactions between pathogen biology, plant development. A thorough understanding of quantitative disease resistance (QDR) would contribute to the design and deployment of durably resistant crop cultivars. However, the molecular mechanisms that control QDR remain poorly understood, largely due to the incomplete and inconsistent nature of the resistance phenotype, which is usually conditioned by many loci of small effect (Jesse Poland et al., 2008). QTL mapping methods for complex traits are challenged by new developments in marker technology, phenotyping platforms and breeding methods. Thus QTL mapping approaches will need to also acknowledge the central roles of QTL by environment interactions (QEI) and QTL by trait interactions in the expression of complex traits (Fred van Eeuwijk et al., 2010). DNA marker technology, QTL theory and statistical methodology for QTL analysis have undergone rapid developments in the past two decades. These concepts and the jargon used by molecular biologists may not be clearly understood by plant breeders and other plant scientists (Collard et al., 2005). Host pattern recognition receptor (HPRR) genes, and defenseresponsive or defense-related genes are important resources for quantitative broad spectrum resistance and durable resistance (Yanjun Kou and Shiping Wang, 2010). Adaptation to the hypersensitive R-genes by fungal and bacterial specialists is quite different from the adaptations to polygenic quantitative resistance. In first case a loss mutation in the avirulence gene results in restoring the pathogenicity. In the latter, exemplified by the cereals/take-all pathosystem, the mutation is due to a gain mutation. Adaptation by a loss mutation is expected to be much easier than adaptation through a gain mutation. This is the main reason for the large difference in durability (Jan Parlevliet).QRLs for which the phenotypic effects on QDR have been verified and for which tightly linked DNA-based markers have been determined can be (and are) used in marker assisted selection (MAS) breeding strategies for improvement of crop germplasm and development of cultivars. 


\section{Introduction}

Many agriculturally important traits such as yield, quality and some form of disease resistance are controlled by many genes (poly genes) and are known as quantitative traits. Identification of the genomic region containing few or more genes controlling these complex traits is a basic idea of QTL mapping. The large number of QTL mapping studies for diverse crop species has provided an abundance of DNA marker-trait associations. The information obtained on the QTL analysis can be utilized for the crop improvement through marker aided selection and molecular breeding. (Veeresha et al., 2015).

Quantitative disease resistance (QDR) has been observed within many crop plants but is not as well understood as qualitative (monogenic) disease resistance and has not been used as extensively in breeding. Mapping quantitative trait loci (QTLs) is a powerful tool for genetic dissection of QDR. DNA markers tightly linked to quantitative resistance loci (QRLs) controlling QDR can be used for marker-assisted selection (MAS) to incorporate these valuable traits. QDR confers a reduction, rather than lack, of disease and has diverse biological and molecular bases as revealed by cloning of QRLs and identification of the candidate gene(s) underlying QRLs. Increasing our biological knowledge of QDR and QRLs will enhance understanding of how QDR differs from qualitative resistance and provide the necessary information to better deploy these resources in breeding. Application of MAS for QRLs in breeding for QDR to diverse pathogens is illustrated by examples from wheat, barley, common bean, tomato, and pepper. Strategies for optimum deployment of QRLs require research to understand effects of QDR on pathogen populations over time.
Quantitative trait locus (QTL) mapping is a highly effective approach for studying genetically complex forms of plant disease resistance. With QTL mapping, the roles of specific resistance loci can be described, racespecificity of partial resistance genes can be assessed, and interactions between resistance genes, plant development, and the environment can be analysed. Outstanding examples include: quantitative resistance to the rice blast fungus, late blight of potato, gray leaf spot of maize, bacterial wilt of tomato, and the soybean cyst nematode. These studies provide insights into the number of quantitative resistance loci involved in complex disease resistance, epistatic and environmental interactions, racespecificity of partial resistance loci, interactions between pathogen biology, plant development and biochemistry, and the relationship between qualitative and quantitative loci. QTL mapping also provides a framework for marker-assisted selection of complex disease resistance characters and the positional cloning of partial resistance genes (Young, 1996).

\section{What are genetic markers?}

Genetic markers represent genetic differences between individual organisms or species. Generally, they do not represent the target genes themselves but act as 'signs' or 'flags'. Genetic markers that are located in close proximity to genes (i.e. tightly linked) may be referred to as gene 'tags'. Such markers themselves do not affect the phenotype of the trait of interest because they are located only near or 'linked' to genes controlling the trait. All genetic markers occupy specific genomic positions within chromosomes (like genes) called 'loci' (singular 'locus').

There are three major types of genetic markers: 
Morphological (also 'classical' or 'visible') markers which themselves are phenotypic traits or characters;

Biochemical markers, which include allelic variants of enzymes called isozymes; and

DNA (or molecular) markers, which reveal sites of variation in DNA (Jones et al., 1997; Winter and Kahl, 1995).

DNA markers are the most widely used type of marker predominantly due to their abundance. They arise from different classes of DNA mutations such as substitution mutations (point mutations), rearrangements (insertions or deletions) or errors in replication of randomly repeated DNA (Paterson, 1996a). These markers are selectively neutral because they are usually located in non-coding regions of DNA. Unlike morphological and biochemical markers, DNA markers are practically unlimited in number and are not affected by environmental factors and/or the developmental stage of the plant (Winter and Kahl, 1995). Apart from the use of DNA markers in the construction of linkage maps, they have numerous applications in plant breeding such as assessing the level of genetic diversity within germplasm and cultivar identity (Baird et al., 1997; Henry, 1997; Jahufer et al., 2003; Weising et al., 1995; Winter and Kahl, 1995). DNA markers may be broadly divided into three classes based on the method of their detection: (1) hybridization-based; (2) polymerase chain reaction (PCR)-based and (3) DNA sequencebased (Gupta et al., 1999; Jones et al., 1997; Joshi et al., 1999; Winter and Kahl, 1995). Essentially, DNA markers may reveal genetic differences that can be visualised by using a technique called gel electrophoresis and staining with chemicals (ethidium bromide or silver) or detection with radioactive or colourimetric probes. DNA markers are particularly useful if they reveal differences between individuals of the same or different species. These markers are called polymorphic markers, whereas markers that do not discriminate between genotypes are called monomorphic markers. Polymorphic markers may also be described as codominant or dominant. This description is based on whether markers can discriminate between homozygotes and heterozygotes.

\section{What are linkage maps?}

A linkage map may be thought of as a 'road map' of the chromosomes derived from two different parents (Paterson, 1996a). Linkage maps indicate the position and relative genetic distances between markers along chromosomes, which is analogous to signs or landmarks along a highway. The most important use for linkage maps is to identify chromosomal locations containing genes and QTLs associated with traits of interest; such maps may then be referred to as 'QTL' (or 'genetic') maps. 'QTL mapping' is based on the principle that genes and markers segregate via chromosome recombination (called crossing-over) during meiosis (i.e. sexual reproduction), thus allowing their analysis in the progeny (Paterson, 1996a). Genes or markers that are close together or tightlylinked will be transmitted together from parent to progeny more frequently than genes or markers that are located further apart. In a segregating population, there is a mixture of parental and recombinant genotypes. The frequency of recombinant genotypes can be used to calculate recombination fractions, which may by used to infer the genetic distance between markers. By analysing the segregation of markers, the relative order and distances between markers can be determined-the lower the frequency of recombination between two markers, the closer they are situated on a chromosome (conversely, the higher the frequency of 
recombination between two markers, the further away they are situated on a chromosome). Markers that have a recombination frequency of $50 \%$ are described as 'unlinked' and assumed to be located far apart on the same chromosome or on different chromosomes. For a more detailed explanation of genetic linkage, the reader is encouraged to consult basic textbooks on genetics or quantitative genetics (for example, Hartl and Jones, 2001; Kearsey and Pooni, 1996). Mapping functions are used to convert recombination fractions into map units called centi- Morgans (cM) (discussed later). Linkage maps are constructed from the analysis of many segregating markers. The three main steps of linkage map construction are: (1) production of a mapping population; (2) identification of polymorphism and (3) linkage analysis of markers.

\section{Mapping populations}

The construction of a linkage map requires a segregating plant population (i.e. a population derived from sexual reproduction). The parents selected for the mapping population will differ for one or more traits of interest. Population sizes used in preliminary genetic mapping studies generally range from 50 to 250 individuals (Mohan et al., 1997). F2 populations, derived from F1 hybrids, and backcross (BC) populations, derived by crossing the F1 hybrid to one of the parents, are the simplest types of mapping populations developed for self-pollinating species. Their main advantages are that they are easy to construct and require only a short time to produce. Inbreeding from individual F2 plants allows the construction of recombinant inbred (RI) lines, which consist of a series of homozygous lines, each containing a unique combination of chromosomal segments from the original parents. The length of time needed for producing RI populations is the major disadvantage, because usually six to eight generations are required. Doubled haploid (DH) populations maybe produced by regenerating plants by the induction of chromosome doubling from pollen grains, however, the production of DH populations is only possible in species that are amenable to tissue culture (e.g. cereal species such as rice, barley and wheat). The major advantages of $\mathrm{RI}$ and DH populations are that they produce homozygous or 'true-breeding' lines that can be multiplied and reproduced without genetic change occurring.

\section{Identification of polymorphism}

It is critical that sufficient polymorphism exists between parents in order to construct a linkage map (Young, 1994). In general, cross pollinating species possess higher levels of DNA polymorphism compared to inbreeding species; mapping in inbreeding species generally requires the selection of parents that are distantly related. Once polymorphic markers have been identified, they must be screened across the entire mapping population, including the parents (and F1 hybrid, if possible).This is known as marker 'genotyping' of the population.

\section{Linkage analysis of markers}

The final step of the construction of a linkage map involves coding data for each DNA marker on each individual of a population and conducting linkage analysis using computer programs. Although linkage analysis can be performed manually for a few markers, it is not feasible to manually analyse and determine linkages between large numbers of markers that are used to construct maps; computer programs are required for this purpose. Linkage between markers is usually calculated using odds ratios (i.e. the ratio of linkage versus no linkage). This ratio is more conveniently expressed as the logarithm of the ratio, and is called a logarithm of odds 
(LOD) value or LOD score (Risch, 1992). LOD values of $>3$ are typically used to construct linkage maps. Commonly used software programs include Mapmaker/ EXP (Lander et al., 1987; Lincoln et al., 1993a) and MapManager QTX (Manly et al., 2001), which are freely available from the internet. JoinMap is another commonly-used program for constructing linkage maps (Stam, 1993). Linked markers are grouped together into 'linkage groups,' which represent chromosomal segments or entire chromosomes. The accuracy of measuring the genetic distance and determining marker order is directly related to the number of individuals studied in the mapping population.

\section{Genetic distance and mapping functions}

Distance along a linkage map is measured in terms of the frequency of recombination between genetic markers (Paterson, 1996a). Mapping functions are required to convert recombination fractions into centi-Morgans (cM) because recombination frequency and the frequency of crossing-over are not linearly related (Hartl and Jones, 2001; Kearsey and Pooni, 1996). When map distances are small $(<10 \mathrm{cM})$, the map distance equals the recombination frequency. However, this relationship does not apply for map distances that are greater than $10 \mathrm{cM}$ (Hartl and Jones, 2001). Two commonly used mapping functions are the Kosambi mapping function, which assumes that recombination events influence the occurrence of adjacent recombination events, and the Haldane mapping function, which assumes no interference between crossover events (Hartl and Jones, 2001; Kearsey and Pooni, 1996). It should be noted that distance on a linkage map is not directly related to the physical distance of DNA between genetic markers, but depends on the genome size of the plant species (Paterson, 1996a). Furthermore, the relationship between genetic and physical distance varies along a chromosome (Kunzel et al., 2000; Tanksley et al., 1992; Young, 1994). For example, there are recombination 'hot spots' and 'cold spots,' which are chromosomal regions in which recombination occurs more frequently or less frequently, respectively (Faris et al., 2000; Ma et al., 2001; Yao et al., 2002).

\section{QTL analysis}

\section{Principle of QTL analysis}

QTL analysis is based on the principle of detecting an association between phenotype and the genotype of markers. Markers are used to partition the mapping population into different genotypic groups based on the presence or absence of a particular marker locus and to determine whether significant differences exist between groups with respect to the trait being measured (Tanksley, 1993; Young, 1996). A significant difference between phenotypic means of the groups (either 2 or 3 ), depending on the marker system and type of population, indicates that the marker locus being used to partition the mapping population is linked to a QTL controlling the trait. The closer a marker is from a QTL, the lower the chance of recombination occurring between marker and QTL. Therefore, the QTL and marker will be usually be inherited together in the progeny, and the mean of the group with the tightlylinked marker will be significantly different. Principle of QTL mapping (adapted from Young, 1996). Markers that are linked to a gene or QTL controlling a particular trait (e.g. plant height) will indicate significant differences when the mapping population is partitioned according to the genotype of the marker. The closer the marker is to the QTL of interest, the lower the chance for recombination between marker and QTL. Therefore, the QTL and marker will be 
usually be inherited together in the progeny, and the mean of the group with the tightlylinked marker will be significantly different $(\mathrm{P}<0.05)$ to the mean of the group without the marker. When a marker is loosely-linked or unlinked to a QTL, there is independent segregation of the marker and QTL.

\section{Methods to detect QTLs}

Three widely-used methods for detecting QTLs are single-marker analysis, simple interval mapping and composite interval mapping (Liu, 1998; Tanksley, 1993). Singlemarker analysis (also single-point analyses) is the simplest method for detecting QTLs associated with single markers. The statistical methods used for single-marker analysis include t-tests, analysis of variance (ANOVA) and linear regression. Linear regression is most commonly used because the coefficient of determination (R2) from the marker explains the phenotypic variation arising from the QTL linked to the marker. This method does not require a complete linkage map and can be performed with basic statistical software programs. Sometimes, the allele size of the marker is also reported. Q -Gene and MapManager QTX are commonly used computer programs to perform single-marker analysis (Manly et al., 2001; Nelson, 1997). The simple interval mapping (SIM) method makes use of linkage maps and analyses intervals between adjacent pairs of linked markers along chromosomes simultaneously, instead of analysing single markers (Lander and Botstein, 1989). The use of linked markers for analysis compensates for recombination between the markers and the QTL, and is considered statistically more powerful compared to single-point analysis (Lander and Botstein, 1989; Liu, 1998). More recently, composite interval mapping (CIM) has become popular for mapping QTLs. This method combines interval mapping with linear regression and includes additional genetic markers in the statistical model in addition to an adjacent pair of linked markers for interval mapping (Jansen, 1993; Jansen and Stam, 1994; Zeng, 1993, 1994). The main advantage of CIM is that it is more precise and effective at mapping QTLs compared to single-point analysis and interval mapping, especially when linked QTLs are involved. Many researchers have used QTL Cartographer (Basten et al., 1994, 2001), MapManager QTX (Manly et al., 2001) and PLABQTL (Utz and Melchinger, 1996) to perform CIM.

\section{Understanding interval mapping results}

QTLs are located with respect to a linkage map. The results of the test statistic for SIM and CIM are typically presented using a logarithmic of odds (LOD) score or likelihood ratio statistic (LRS). There is a direct one-toone transformation between LOD scores and LRS scores (the conversion can be calculated by: $\mathrm{LRS}=4.6 \times$ LOD) (Liu, 1998). These LOD or LRS profiles are used to identify the most likely position for a QTL in relation to the linkage map, which is the position where the highest LOD value is obtained. A typical output from interval mapping is a graph with markers comprising linkage groups on the $\mathrm{x}$ axis and the test statistic on the y axis. Before permutation tests were widely accepted as an appropriate method to determine significance thresholds, a LOD score of between 2.0 to 3.0 (most commonly 3.0) was usually chosen as the significance threshold.

\section{Reporting and describing QTLs detected from interval mapping}

The most common way of reporting QTLs is by indicating the most closely linked markers in a table and/or as bars (or oval shapes or arrows) on linkage maps (for example: Beattie et al., 2003; George et al., 2003; Hittalmani et al., 2002; Jampatong et al., 2002; Mc Couch and Doerge, 1995; PiletNayel et al., 2002), the reason for reporting 
QTL mapping of height and disease resistance traits. Hypothetical QTLs were detected on chromosomes 2, 3, 4 and 5. A major QTL for height was detected on chromosome 2. Two major QTLs for disease resistance were detected on chromosomes 4 and 5 whereas a minor QTL was detected on chromosome 3 (adapted from Hartl and Jones, 2001). Flanking markers is that selection based on two markers should be more reliable than selection based on a single marker. In general terms, an individual QTL may also be described as 'major' or 'minor'. This definition is based on the proportion of the phenotypic variation explained by a QTL (based on the R2 value): major QTLs will account for a relatively large amount (e.g. $>10 \%$ ) and minor QTLs will usually account for $<10 \%$. Sometimes, major QTLs may refer to QTLs that are stable across environments whereas minor QTLs may refer to QTLs that may be environmentally sensitive, especially for QTLs that are associated with disease resistance (Li et al., 2001; Lindhout, 2002; Pilet-Nayel et al., 2002). In more formal terms, QTLs may be classified as: (1) suggestive; (2) significant; and (3) highly significant (Lander and Kruglyak, 1995). Lander and Kruglyak (1995) proposed this classification in order to "avoid a flood of false positive claims" and also ensure that "true hints of linkage" were not missed. Significant and highly-significant QTLs were given significance levels of 5 and $0.1 \%$, respectively, whereas a suggestive QTL is one that would be expected to occur once at random in a QTL mapping study (in other words, there is a warning regarding the reliability of suggestive QTLs). The mapping program MapManager QTX reports QTL mapping results with this classification (Manly et al., 2001).

\section{Confidence intervals for QTLs}

Although the most likely position of a QTL is the map position at which the highest LOD or
LRS score is detected, QTLs actually occur within confidence intervals. There are several ways in which confidence intervals can be calculated. The simplest is the 'one-LOD support interval,' which is determined by finding the region on both sides of a QTL peak that corresponds to a decrease of 1 LOD score (Hackett, 2002; Lander and Botstein, 1989). 'Bootstrapping' - a statistical method for resampling-is another method to determine the confidence interval of QTLs (Liu, 1998; Visscher et al., 1996), and can be easily applied within some mapping software programs such as MapManager QTX (Manly et al., 2001).

\section{Factors influencing the detection of QTLs}

There are many factors that influence the detection of QTLs segregating in a population (Asins, 2002; Tanksley, 1993). The main ones are genetic properties of QTLs that control traits, environmental effects, population size and experimental error.

QTLs that are closely-linked (approximately $20 \mathrm{cM}$ or less) will usually be detected as a single QTL in typical population sizes $(<500)$ (Tanksley, 1993) Environmental effects may have a profound influence on the expression of quantitative traits. Experiments that are replicated across sites and over time (e.g. different seasons and years) may enable the researcher to investigate environmental influences o Environmental effects may have a profound influence on the expression of quantitative traits, experiments that are replicated across sites and over time (e.g. different seasons and years) may enable the researcher to investigate environmental influences on QTLs affecting trait(s) of interest (George et al., 2003; Hittalmani et al., 2002; Jampatong et al., 2002; Lindhout, 2002; Paterson et al., 1991b; Price and Courtois, 1999). As discussed previously, RI or $\mathrm{DH}$ populations are ideal for these purposes. The most important experimental design factor is 
the size of the population used in the mapping study. The larger the population, the more accurate the mapping study and the more likely it is to allow detection of QTLs with smaller effects (Haley and Andersson, 1997; Tanksley, 1993). An increase in population size provides gains in statistical power, estimates of gene effects and confidence intervals of the locations of QTLs (Beavis, 1998; Darvasi et al., 1993). The main sources of experimental error are mistakes in marker genotyping and errors in phenotypic evaluation. Genotyping errors and missing data can affect the order and distance between markers within linkage maps (Hackett, 2002). The accuracy of phenotypic evaluation is of the utmost importance for the accuracy of QTL mapping. A reliable QTL map can only be produced from reliable phenotypic data. Replicated phenotypic measurements or the use of clones (via cuttings) can be used to improve the accuracy of QTL mapping by reducing background 'noise' (Danesh et al., 1994; Haley and Andersson, 1997). Some thorough studies include those where phenotypic evaluations have been conducted in both field and glasshouse trials, for ascochyta blight resistance in chickpea (Flandez- Galvez et al., 2003a), bacterial brown spot in common bean (Jung et al., 2003), and downy mildew resistance in pearl millet (Jones et al., 2002). Confirmation of QTL mapping ideally, due to the factors described above, QTL mapping studies should be independently confirmed or verified (Lander and Kruglyak, 1995). Such confirmation studies (referred to as 'replication studies' by Lander and Kruglyak, 1995) may involve independent populations constructed from the same parental genotypes or closely-related genotypes used in the primary QTL mapping study. Sometimes, larger population sizes may be used. Furthermore, some recent studies have proposed that QTL positions and effects should be evaluated in independent populations, because QTL mapping based on typical population sizes results in a low power of QTL detection and a large bias of QTL effects (Melchinger et al., 1998; Utz et al., 2000). Unfortunately, due to constraints such as lack of research funding and time, and possibly a lack of understanding of the need to confirm results, QTL mapping studies are rarely confirmed. Some notable exceptions are the confirmation of QTLs associated with root-knot nematode resistance (Li et al., 2001) and bud blight resistance in soybean (Fasoula et al., 2003) QTL affecting trait(s) of interest (George et al., 2003; Hittalmani et al., 2002; Jampatong et al., 2002; Lindhout, 2002; Paterson et al., 1991b; Price and Courtois, 1999). As discussed previously, RI or DH populations are ideal for these purposes. The most important experimental design factor is the size of the population used in the mapping study. The larger the population, the more accurate the mapping study and the more likely it is to allow detection of QTLs with smaller effects (Haley and Andersson, 1997; Tanksley, 1993). An increase in population size provides mains in statistical power, estimates of gene effects and confidence intervals of the locations of QTLs (Beavis, 1998; Darvasi et al., 1993). The main sources of experimental error are mistakes in marker genotyping and errors in phenotypic evaluation. Genotyping errors and missing data can affect the order and distance between markers within linkage maps (Hackett, 2002). The accuracy of phenotypic evaluation is of the utmost importance for the accuracy of QTL mapping. A reliable QTL map can only be produced from reliable phenotypic data. Replicated phenotypic measurements or the use of clones (via cuttings) can be used to improve the accuracy of QTL mapping by reducing background 'noise' (Danesh et al., 1994; Haley and Andersson, 1997). Some thorough studies include those where phenotypic evaluations have been conducted in both field and glasshouse trials, for 
ascochyta blight resistance in chickpea (Flandez- Galvez et al., 2003a), bacterial brown spot in common bean (Jung et al., 2003), and downy mildew resistance in pearl millet (Jones et al., 2002).

\section{Confirmation of QTL mapping}

Ideally, due to the factors described above, QTL mapping studies should be independently confirmed or verified (Lander and Kruglyak, 1995). Such confirmation studies (referred to as 'replication studies' by Lander and Kruglyak, 1995) may involve independent populations constructed from the same parental genotypes or closely-related genotypes used in the primary QTL mapping study. Sometimes, larger population sizes may be used. Furthermore, some recent studies have proposed that QTL positions and effects should be evaluated in independent populations, because QTL mapping based on typical population sizes results in a low power of QTL detection and a large bias of QTL effects (Melchinger et al., 1998; Utz et al., 2000). Unfortunately, due to constraints such as lack of research funding and time, and possibly a lack of understanding of the need to confirm results, QTL mapping studies are rarely confirmed. Some notable exceptions are the confirmation of QTLs associated with root-knot nematode resistance ( $\mathrm{Li}$ et al., 2001) and bud blight resistance in Soybean (Fasoula et al., 2003). Another approach used to confirm QTLs has been to use a specific type of population called near isogenic lines (NILs).

\section{Towards marker-assisted selection}

Marker-assisted selection (MAS) is a method whereby a phenotype is selected on the genotype of a marker.

\section{High-resolution mapping of QTLs}

The preliminary aim of QTL mapping is to produce a comprehensive 'framework' (also 'skeletal' or 'scaffold' linkage map) that covers all chromosomes evenly in order to identify markers flanking those QTLs that control traits of interest. There are several more steps required, because even the closest markers flanking a QTL may not be tightly linked to a gene of interest (Michelmore, 1995). This means that recombination can occur between a marker and QTL, thus reducing the reliability and usefulness of the marker. By using larger population sizes and a greater number of markers, more tightlylinked markers can be identified; this process is termed 'high-resolution mapping' (also 'fine mapping'). Therefore, high-resolution mapping of QTLs may be used to develop reliable markers for marker assisted selection (at least $<5 \mathrm{cM}$ but ideally $<1 \mathrm{cM}$ away from the gene) and also to discriminate between a single gene or several linked genes (Michelmore, 1995; Mohan et al., 1997). High-resolution maps of specific chromosomal regions may also be constructed by using NILs (Blair et al., 2003).

\section{Validation of markers}

Generally, markers should be validated by testing their effectiveness in determining the target phenotype in independent populations and different genetic backgrounds, which is referred to as 'marker validation' (Cakir et al., 2003; Collins et al., 2003; Jung et al., 1999; Langridge et al., 2001; Li et al., 2001; Sharp et al., 2001).

\section{Marker-assisted selection}

Selecting plants in a segregating progeny that contain appropriate combinations of genes is a critical component of plant breeding (Ribaut and Betran, 1999; Weeden et al., 1994). Moreover, plant breeders typically work with hundreds or even thousands of populations, which often contain large numbers (Ribaut and Betran, 1999; Witcombe and Virk, 2001). 
'Marker-assisted selection' (also 'markerassisted breeding' or 'marker-aided selection') may greatly increase the efficiency and effectiveness in plant breeding compared to conventional breeding methods.

\section{The advantages of MAS include}

1. Time saving from the substitution of complex field trials (that need to be conducted at particular times of year or at specific locations, or are technically complicated) with molecular tests;

2. Elimination of unreliable phenotypic evaluation associated with field trials due to environmental effects;

3. Selection of genotypes at seedling stage;

4. Gene 'pyramiding' or combining multiple genes simultaneously;

5. Avoid the transfer of undesirable or deleterious genes ('linkage drag'; this is of particular relevance when the introgression of genes from wild species is involved).

6. Selecting for traits with low heritability.

7. Testing for specific traits where phenotypic evaluation is not feasible (e.g. quarantine restrictions may prevent exotic pathogens to be used for screening).

Marker Assisted Selection for Quantitative Resistance Loci and its application in Quantitative Disease Resistance

\section{A. MAS breeding for individual QRLs}

In case of Fusarium head blight in wheat, on chromosome 3B a major QRL "Fhb1" from cultivar Sumai 3 reduction in disease severity by $23 \%-27 \%$ (Pumphery et al., 2007). Late blight in tomato, QRL on chromosomes 5 and 11 are used (Brouwer and St. Clair, 2004), leaf rust of barley, Rphq2, Rphq3, Rphq4 from variety Vada (Marcel et al., 2007). White mold in common bean, Major QTL located on LGs B2, B7 and B8 of which LGsB7 and B8 reduced disease severity 15\% and $17 \%$ respectively (Miklas et al., 2006).

\section{B. MAS breeding for combining multiple QRLS}

In case of common bacterial blight in common bean (X. axonopodis pv. phaseoli), Combining QRLs on LGs B6+ B8+B10 in Chase cultivar. (Kelly et al., 2003). Root and stem rot in peeper: (Phytophthora capsici), QRLs on chromosomes P5+ P10 transferred from cultiver perennial to yellow wonder. (Thabuis et al., 2004)

\section{MAS breeding for combining QRLs and qualitative resistance gene}

In case of stripe rust in barley (Puccinia striiformis f. sp. hordei), Rpsx (on chromosome 1) + three QRLs (on chromosome 4, 5 and 7) (Castro et al., 2003). Bean golden mosaic virus (BGMV) in common bean, QRL located at linkage group B4 + qualitative resistance gene "bgm-1" (Miklas et al., 2007). PVY and pepper, Two QRLs located at chromosome + qualitative resistance gene "pvr23" (Palloix et al., 2009)

Quantitative disease resistance and quantitative resistance locus

\section{Biological bases}

In recent years, a plethora of papers have been published on mapping QTLs for many traits in crop plants, including resistance to various pathogens. But what do we know about the biology of QRLs? An excellent review by Poland et al., highlights current hypotheses and cites some supporting data for diverse biological bases of QDR and QRLs; an earlier review noted some of the same hypotheses. Hypotheses listed by Poland et al., include: QDR is based on genes regulating 
morphology and developmental traits; QRLs represent mutations or alleles at genes for basal defense; QRLs affect production of components of chemical warfare; QRLs are involved in defense signal transduction; QRLs are weak or defeated R-genes; and QRLs are unidentified genes. The authors noted that no single hypothesis accounts for all QDR and QRLs, and multiple hypotheses for QDR are likely valid. Some QRLs have been reported to be coincident (i.e., have similar or identical linkage map positions) with major R-genes for disease resistances in various crop plants, leading to the hypothesis that QRLs may be weak or defeated R-genes When evolutionary genetic changes in pathogens erode the ability of R-genes to confer complete resistance, defeated R-genes may be detected as QRLs that are associated with a reduction in disease.

\section{Choice of models for QTL mapping with multiple families}

QTL mapping has recently shifted from analysis of single families to multiple, connected families and several biometric models have been suggested. Linkage mapping with individual bi-parental families derived from divergent parent lines has become routine for dissecting the genetic architecture of complex traits in crops (Holland 2007), although it has certain drawbacks. (i) The mapping population originates from two parent lines and, therefore, represents only a small cross section of the breeding germplasm (Xu 1998; Liu and Zeng 2000). (ii) Mapping results from one family are often not transferable to other families (Beavis 1998; Melchinger et al., 1998), because the expression of QTL depends on its presence and in addition can be influenced by the genetic background. To overcome these limitations, multi-family QTL mapping has been proposed to detect QTL jointly from multiple bi-parental families (Jansen et al., 2003; Blanc et al., 2006; Bink et al., 2012). These populations can be either families routinely generated in practical breeding programs (Bardol et al., 2013) or created using special mating designs, e.g., the diallel design (Blanc et al., 2006), nested association mapping population (NAM, Yu et $a l ., 2008$ ) or multiparent advanced generation intercross (MAGIC, Huang et al., 2015). The main difference between bi-parental and multi-family QTL mapping concerns the set of QTL that segregate in one versus several populations, which enables testing for QTL $\times$ genetic background interactions in the latter case (Blanc et al., 2006). For a given sample size, the former approach has generally higher power to detect rare QTL with large effects, segregating in only one or a small number of families, while the latter approach has higher chances to detect common QTL with small effects shared by a large number of families (Li et al., 2011; Ogut et al., 2015).

Four main categories of biometrical models have been developed for multi-family QTL mapping in plants, which differ in their assumptions about the QTL effects: (1) effects are specific to each population (e.g., FULL model in Jannink and Jansen 2001; disconnected model in Blanc et al., 2006), (2) effects of parental alleles are identical over populations (e.g., REDUCED model in Jannink and Jansen 2001; connected model in Blanc et al., 2006), (3) identical by descent (IBD) segments shared by parents have the same alleles, the effects of which are identically expressed in different populations (e.g., HaploMQM- model in Jansen et al., 2003; LDLA model in Bardol et al., 2013 and Giraud et al., 2014), (4) identical by state (IBS) segments among parents harbor identical alleles with effects consistent across genetic backgrounds (Yu et al., 2008; LDLA1-marker model in Bardol et al., 2013; Model-B in Würschum et al., 2012). From category (1) to (4), the number of alleles at QTL decreases, resulting in a reduced number 
of parameters to be estimated. Thus, the power of QTL detection may increase and estimation error of QTL effects may decrease (Rebai and Goffinet 1993, 2000), if a common set of QTL can be assumed. In experimental studies, the performance ranking of these models varied among populations of equal size and among traits (Blanc et al., 2006; Steinhoff et al., 2011; Bardol et al., 2013; Giraud et al., 2014). Therefore, further research is warranted to compare these models and provide guidance for their choice.

In recent years, genomic prediction of breeding values of untested genotypes with genome-wide markers has received considerable interest by breeders due to a dramatic reduction in the costs of genotyping (Meuwissen et al., 2001; Jannink et al., 2010). One important question in genomic prediction, and generally in marker-based prediction, is how to design the training set (TS) for achieving high prediction accuracy. Major factors identified are the sample size and number of families in the TS and their relatedness to the validation set (VS, Riedelsheimer et al., 2013; Lehermeier et al., 2014). Multi-family QTL mapping offers the possibility to unveil the genetic basis of prediction accuracy in genomic prediction with different composition of the TS. (Han et al 2016)

\section{Conclusions of the study are}

1. Quantitative disease resistances (QDRs) can be dissected with the genetic analysis method of quantitative trait locus (QTL) mapping to localize quantitative resistance loci (QRL), which are regions of the host plant genome significantly associated with quantitative variation in disease resistance phenotypes.

2. A QRL contains causal gene(s) and causal quantitative trait nucleotides (QTN) that are responsible for the phenotypic effect on QDR.
3. The biological and molecular bases of QRLs appear to be diverse, based on QRLs that have been cloned and functionally validated to date, as well as QRLs for which candidate genes have been identified and for which corroborative evidence for their role in QDR has been obtained.

4. Although none of the QRLs characterized to date has a gene structure similar to qualitative resistance R-genes or encodes Rgene type proteins with LRR motifs, it is certainly possible that future studies will reveal that some QRLs have similarities to Rgene structures and proteins.

5. QRLs for which the phenotypic effects on QDR have been verified and for which tightly linked DNA-based markers have been determined can be (and are) used in marker assisted selection (MAS) breeding strategies for improvement of crop germplasm and development of cultivars.

\section{Path ahead}

1. There are multiple steps between genotype and phenotype and given that genes interact in networks that include regulatory (non protein coding) regions, there is still much to learn about quantitative trait expression and regulation.

2. How the environment influences quantitative traits on a molecular basis it is still unknown.

3. Do QRLs serve as a back-up system to Rgenes in providing some level of resistance to the crop in the event of R-gene breakdown?

4. New approaches and methods to facilitate reliable and precise high-throughput phenotyping for quantitative traits including QDR are needed. 


\section{References}

Asins, M. 2002. Present and future of quantitative trait locus analysis in plant breeding. Plant Breed, 121: 281-291.

Baird, V., A. Abbott, R. Ballard, B. Sosinski and S. Rajapakse, 1997. DNA Diagnostics in Horticulture, p. 111-130 In: P. Gresshoff (Ed.), Current Topics in Plant Molecular Biology: Technology Transfer of Plant Biotechnology. CRC Press, Boca Raton.

Bardol, N., Ventelon, M., Mangin, B., et al. 2013. Combined linkage and linkage disequilibrium QTL mapping in multiple families of maize (Zea mays L.) line crosses highlights complementarities between models based on parental haplotype and single locus polymorphism. Theor. Appl. Genet., 126: 2717-2736. doi:10.1007/ s00122-013-2167-9

Basten, C.J., B.S. Weir and Z.B. Zeng, 1994. Zmap-a QTL cartographer. In: J.S.G.C. Smith, B.J. Benkel, W.F. Chesnais, J.P. Gibson, B.W. Kennedy and E.B. Burnside (Eds.), Proceedings of the $5^{\text {th }}$ World Congress on Genetics Applied to Livestock Production: Computing Strategies and Software, Guelph, Ontario, Canada. Published by the Organizing Committee, 5th World Congress on Genetics Applied to Livestock Production.

Beattie, A., J. Larsen, T. Michaels and K. Pauls, 2003. Mapping quantitativetrait loci for a common bean (Phaseolus vulgaris L.) ideotype. Genome, 46: 411-422.

Beavis, W. 1998. QTL Analyses: Power, Precision and Accuracy. In: A.H. Paterson (Ed.), Molecular Dissection of Complex Traits. CRC Press, Boca Raton.
Bink, M.C.A.M., Totir, L.R., ter Braak, C.J.F. et al. 2012. QTL linkage analysis of connected populations using ancestral marker and pedigree information. Theor. Appl. Genet., 124: 1097-1113. doi:10.1007/s00122-011-1772-8.

Blair, M., A. Garris, A. Iyer, B. Chapman, S. Kresovich and S. Mc- Couch, 2003. High resolution genetic mapping and candidate gene identification at the $x a 5$ locus for bacterial blight resistance in rice (Oryza sativa L.). Theor. Appl. Genet., 107: 62-73.

Blanc, G., Charcosset, A., Mangin, B., et al. 2006. Connected populations for detecting quantitative trait loci and testing for epistasis: an application in maize. Theor. Appl. Genet., 113:206224. doi:10.1007/s00122-006-0287-1.

Cakir, M., S. Gupta, G.J. Platz, G.A. Ablett, R. Loughman, L.C. Emebiri, D Poulsen, C.D. Li, R.C.M. Lance, N.W. Galwey, M.G.K. Jones and R. Appels, 2003. Mapping and validation of the genes for resistance to Pyrenophora teres f. teres in barley (Hordeum vulgare L.). Aust. J. Agric. Res., 54: 1369-1377.

Collard, B.C.Y., Jahufer, M.Z.Z., Brouwer, J.B., Pang, E.C.K. 2005. An introduction to markers, quantitative trait loci (QTL) mapping and markerassisted selection for crop improvement: the basic concepts. Euphytica, 142: 169-96.

Collard, B.C.Y., E.C.K. Pang and P.W.J. Taylor, 2003. Selection of wild Cicer accessions for the generation of mapping populations segregating for resistance to ascochyta blight. Euphytica, 130: 1-9.

Collins, H.M., J.F. Panozzo, S.J. Logue, S.P. Jefferies and A.R. Barr, 2003. Mapping and validation of chromosome regions associated with high malt extract in barley (Hordeum vulgare L.). Aust. J. 
Agric. Res., 54: 1223-1240.

Danesh, D., S. Aarons, G. McGill and N. Young, 1994. Genetic dissection of oligogenic resistance to bacterial wilt in tomato. Mol. Plant-Microbe Interact., 7: 464-471.

Darvasi, A., A. Weinreb, V. Minke, J.I. Weller and M. Soller, 1993. Detecting marker-QTL linkage and estimating QTL gene effect and map location using a saturated genetic map. Genetics, 134: 943-951.

Dina A. St. Clair. 2010. Quantitative Disease Resistance and Quantitative Resistance Loci in Breeding. Annu. Rev. Phytopathol., 48: 247-268.

Faris, J.D., K.M. Haen and B.S. Gill, 2000. Saturation mapping of a gene-rich recombination hot spot region in wheat. Genetics, 154: 823-835.

Fasoula, V.A., D.K. Harris, M.A. Bailey, D.V. Phillips and H.R. Boerma, 2003. Identification, mapping, and confirmation of a soybean gene for bud blight resistance. Crop Sci., 43: 17541759.

Flandez-Galvez, H., P.K. Ades, R. Ford, E.C.K. Pang and P.W.J.Taylor 2003a. QTL analysis for ascochyta blight resistance in an intraspecific population of chickpea (Cicer arietinum L.). Theor. Appl. Genet., 107: 1257-1265.

Fred, A., van Eeuwijk, Marco, C.A.M., Bink, Karine Chenu and Scott, C., Chapman. 2010. Curr. Opinion in Plant Biol., 13: 193-205.

George, M.L.C., B.M. Prasanna, R.S. Rathore, T.A.S. Setty, F. Kasim, M. Azrai, S. Vasal, O. Balla, D. Hautea, A. Canama, E. Regalado, M. Vargas, M. Khairallah, D. Jeffers and D. Hoisington, 2003. Identification of QTLs conferring resistance to downy mildews of maize in Asia. Theor. Appl. Genet., 107: 544-551.

Giraud, H., Lehermeier, C., Bauer, E., et al.
2014. Linkage disequilibrium with linkage analysis of multiline crosses reveals different multiallelic QTL for hybrid performance in the flint and dent heterotic groups of maize. Genetics, 198: 1717-1734. doi:10.1534/ genetics.114.169367.

Gupta, P., R. Varshney, P. Sharma and B. Ramesh, 1999. Molecular markers and their applications in wheat breeding. Plant Breed, 118: 369-390.

Hackett, C. 2002. Statistical methods for QTL mapping in cereals. Plant Mol. Biol., 48: 585-599.

Haley, C. and L. Andersson, 1997. Linkage mapping of quantitative trait loci in plants and animals, pp. 49-71. In: P. Dear (Ed.),Genome mapping-A practical approach, Oxford University Press, New York.

Han, S., Friedrich, H. U., Michael Stange, S. M., Würschum, T., Miedaner, T., Bauer, E., Schön, C. C., Melchinger, A. E. 2016. Choice of models for QTL mapping with multiple families and design of the training set for prediction of Fusarium resistance traits in maize. Theor. Appl. Genet., 129: 431-444.

Hartl, D. and E. Jones. 2001. Genetics: Analysis of Genes and Genomes, Jones and Bartlett Publishers, Sudbury, MA.

Henry, R. 1997. Molecular markers in plant improvement, In: Practical Applications of Plant Molecular Biology, pp. 99132, Chapman and Hall, London.

Hittalmani, S., H.E. Shashidhar, P.G. Bagali, N. Huang, J.S. Sidhu, V.P. Singh and G.S. Khush. 2002. Molecular mapping of quantitative trait loci for plant growth, yield and yield related traits across three diverse locations in a doubled haploid rice population. Euphytica, 125: 207-214.

Hori, K., T. Kobayashi, A. Shimizu, K. Sato, K. Takeda and S. Holland, J.B. 2007. Genetic architecture of complex traits in 
plants. Curr. Opin. Plant Biol., 10: 156-161. doi:10.1016/j. pbi.2007.01.003.

Huang, B.E., Verbyla, K.L., Verbyla, A.P. et al. 2015. MAGIC populations in crops: current status and future prospects. Theor. Appl. Genet., 128: 999-1017.

Jahufer, M., B. Barret, A. Griffiths and D. Woodfield, 2003. DNA fingerprinting and genetic relationships among white clover cultivars. In: J. Morton (Ed.), Proceedings of the New Zealand Grassland Association, Vol. 65, pp. 163-169, Taieri Print Limited, Dunedin.

Jampatong, C., M. McMullen, B. Barry, L. Darrah, P. Byrne and H. Kross, 2002. Quantitative trait loci for first- and secondgeneration European corn borer resistance derived from maize inbred Mo47. Crop Sci., 42: 584-593.

Jannink, J.L., Jansen, R. 2001. Mapping epistatic quantitative trait loci with onedimensional genome searches. Genetics, 157: 445-454.

Jannink, J.L., Lorenz, A.J., Iwata, H. 2010. Genomic selection in plant breeding: from theory to practice. Brief Funct. Genom., $\quad$ 9: 166-177. doi:10.1093/bfgp/elq001.

Jansen, R.C., Jannink, J.L., Beavis, W.D. 2003. Mapping quantitative trait loci in plant breeding populations. Crop Sci., 43: 829 . doi:10.2135/ cropsci2003.0829.

Jansen, R. and P. Stam. 1994. High resolution of quantitative traits into multiple loci via interval mapping. Genetics 136: 1447-1455.

Jansen, R., 1993. Interval mapping of multiple quantitative trait loci. Genetics, 135: 205-211.

Jones, E.S., W.A. Breese, C.J. Liu, S.D. Singh, D.S. Shaw and J.R. Witcombe, 2002. Mapping quantitative trait loci for resistance to downy mildewin pearl millet: Field and glasshouse screens detect the same QTL. Crop Sci., 42: 1316-1323.

Jones, N., H. Ougham and H. Thomas, 1997. Markers and mapping: We are all geneticists now. New Phytol., 137: 165177.

Joshi, S., P. Ranjekar and V. Gupta, 1999. Molecular markers in plant genome analysis. Curr. Sci., 77: 230-240.

Jung, G., P.W. Skroch, J. Nienhuis, D.P. Coyne, E. Arnaud-Santana, H.M. Ariyarathne and J.M. Marita, 1999. Confirmation of QTL associated with common bacterial blight resistance in four different genetic backgrounds in common bean. Crop Sci., 39: 14481455.

Kearsey, M. and H. Pooni, 1996. The genetical analysis of quantitative traits. Chapman and Hall, London. Kelly, J.D. and P.N. Miklas, 1998. The role of RAPD markers in breeding for disease resistance in common bean. Mol. Breed, 4: 1-11.

Kelly, J.D., P. Gepts, P.N. Miklas and D.P. Coyne. 2003. Tagging and mapping of genes and QTL and molecular markerassisted selection for traits of economic importance in bean and cowpea. Field Crops Res., 82: 135-154.

Kunzel, G., L. Korzun and A. Meister, 2000. Cytologically integrated physical restriction fragment length polymorphism maps for the barley genome based on translocation breakpoints. Genetics, 154: 397-412.

Lander, E. and D. Botstein, 1989. Mapping Mendelian factors underlying quantitative traits using RFLP linkage maps. Genetics, 121: 185-199.

Lander, E.S. and L. Kruglyak, 1995. Genetic dissection of complex traits: Guidelines for interpreting and reporting linkage results. Nat. Genet., 11: 241-247.

Lander, E.S., P. Green, J. Abrahamson, A. 
Barlow, M.J. Daly, S.E. Lincoln and L. Newburg, 1987. Mapmaker an interactive computer package for constructing primary genetic linkage maps of experimental and natural populations. Genomics, 1: 174-181.

Lehermeier, C., Krämer, N., Bauer, E., et al. 2014. Usefulness of multi-parental populations of maize (Zea mays L.) for genome-based prediction. Genetics, 198:3-16.doi:10.1534/Genetics.

114.161943.

Li, H., Bradbury, P., Ersoz, E., et al. 2011. Joint QTL linkage mapping for multiple-cross mating design sharing one common parent. PLoS One, doi:10.1371/journal.pone.0017573.

Lincoln, S., M. Daly and E. Lander, 1993a. Constructing genetic linkage maps with MAPMAKER/EXP. Version 3.0. Whitehead Institute for Biomedical Research Technical Report, 3rd Edn.

Lincoln, S., M. Daly and E. Lander, 1993b. Mapping genes controlling quantitative traits using MAPMAKER/QTL. Version 1.1. Whitehead Institute for Biomedical Research Technical Report, $2^{\text {nd }}$ Edn.

Lindhout, P., 2002. The perspectives of polygenic resistance in breeding for durable disease resistance. Euphytica, 124: 217-226.

Liu, Y., Zeng, Z.B. 2000. A general mixture model approach for mapping quantitative trait loci from diverse cross designs involving multiple inbred lines. Genet. Res., 75: 345-355. doi:10.1017/ S0016672300004493.

Liu, B., 1998. Statistical Genomics: Linkage, Mapping and QTL Analysis CRC Press, Boca Raton.

Ma, X.F., K. Ross and J.P. Gustafson. 2001. Physical mapping of restriction fragment length polymorphism (RFLP) markers in homoeologous groups 1 and 3 chromosomes of wheat by in situ hybridization. Genome, 44: 401-412.

Manly, K.F., H. Cudmore Robert, Jr. and J.M. Meer, 2001. Map Manager QTX, crossplatform software for genetic mapping. Mamm Genome, 12: 930-932.

McCouch, S.R. and R.W. Doerge, 1995. QTL mapping in rice. Trends Genet., 11: 482-487.

McCouch, S.R., X. Chen, O. Panaud, S. Temnykh, Y. Xu, Y. Cho, N. Huang, T. Ishii and M. Blair, 1997. Microsatellite marker development, mapping and applications in rice Genetics, and breeding. Plant Mol. Biol., 35: 89-99.

Melchinger, A.E., H.F. Utz and C.C. Schon, 1998. Quantitative trait locus (QTL) mapping using different testers and independent population samples in maize reveals low power of QTL detection and large bias in estimates of QTL effects. Genetics, 149: 383-403.

Meuwissen, T.H., Hayes, B.J., Goddard, M.E. 2001. Prediction of total genetic value using genome-wide dense marker maps. Genetics, 157:1819-1829.

Mohan, M., S. Nair, A. Bhagwat, T.G. Krishna, M.Yano, C.R. Bhatia and T. Sasaki, 1997. Genome mapping, molecular markers and marker-assisted selection in crop plants. Mol. Breed, 3: 87-103.

Nelson, J.C. 1997. Qgene-software for marker-based genomic analysis and breeding. Mol. Breed, 3: 239-245.

Ogut, F., Bian, Y., Bradbury, P.J., Holland, J.B. 2015. Joint-multiple family linkage analysis predicts within-family variation better than single-family analysis of the maize nested association mapping population. Hered (Edinb), 114: 552-563. doi:10.1038/ hdy.2014.123.

Parlevliet, J.E. 2002. Durability of resistance against fungal, bacterial and viral pathogens: present situation. Euphytica, 124: 147-56. 
Paterson, A.H., 1996a. Making genetic maps. In: A.H. Paterson (Ed.), Genome Mapping in Plants, pp. 23-39. R. G. Landes Company, San Diego, California; Academic Press, Austin, Texas.

Paterson, A.H., S. Damon, J.D. Hewitt, D. Zamir, H.D. Rabinowitch, S.E. Lincoln, E.S. Lander and S.D. Tanksley, 1991 b. Mendelian factors underlying quantitative traits in tomato comparison across species generations and environments. Genetics, 127: 181-198.

Pilet-Nayel, M.L., F.J. Muehlbauer, R.J. McGee, J.M. Kraft, A. Baranger and C.J. Coyne, 2002. Quantitative trait loci for partial resistance to Aphanomyces root rot in pea. Theor. Appl. Genet., 106: 28-39.

Poland, J.A., Balint-Kurti, P.J., Wisser, R.J., Pratt, R.C., Nelson, R.J. 2008. Shades of gray: the world of quantitative disease resistance. Trends Plant Sci., 14: 21-29.

Price, A. and B. Courtois, 1999. Mapping QTLs associated with drought resistance in rice: Progress, problems and prospects. Plant Growth Reg., 29: 123-133.

Rebai, A., Goffinet, B. 1993. Power of tests for QTL detection using replicated progenies derived from a diallel cross. Theor. Appl. Genet., 86:1014-1022. doi:10.1007/BF00211055.

Rebai, A., Goffinet, B. 2000. More about quantitative trait locus mapping with diallel designs. Genet. Res., 75: 243247.

Ribaut, J.M. and J. Betran, 1999. Single largescale marker-assisted selection (SLSMAS). Mol. Breed, 5: 531-541.

Riedelsheimer, C., Endelman, J.B., Stange, M., et al. 2013. Genomic predictability of interconnected biparental maize populations. Genetics, 194:493-503. doi:10.1534/Genetics,.113.150227.
Risch, N. 1992. Genetic linkage: Interpreting LOD scores. Sci., 255: 803-804.

Sharp, P.J., S. Johnston, G. Brown, R.A. McIntosh, M. Pallotta, M. Carter, H.S. Bariana, S. Khartkar, E.S. Lagudah, R.P. Singh, M. Khairallah, R. Potter and M.G.K. Jones, 2001. Validation of molecular markers for wheat breeding. Aust. J. Agric. Res., 52: 1357- 1366.

Stam, P. 1993. Construction of integrated genetic linkage maps by means of a new computer package: Join Map. Plant J., 3: 739-744.

Steinhoff, J., Liu, W., Maurer, H.P. et al. 2011. Multiple-line cross quantitative trait locus mapping in European elite maize. Crop Sci., 51:2505. doi:10.2135/cropsci2011.03.0181.

Tanksley, S.D. 1993. Mapping polygenes. Annu. Rev. Genet., 27: 205- 233.

Tanksley, S.D., M.W. Ganal, J.P. Prince, M.C. De Vicente, M.W. Bonierbale, P. Broun, T.M. Fulton, J.J. Giovannoni and S. Grandillo. 1992. High density molecular linkage maps of the tomato and potato genomes. Genetics, 132: 1141-1160.

Utz, H. and A. Melchinger, 1996. PLABQTL: A program for composite interval mapping of QTL. J. Quant. Trait Loci., 2(1).

Utz, H.F., A.E. Melchinger and C.C. Schon. 2000. Bias and sampling error of the estimated proportion of genotypic variance explained by quantitative trait loci determined from experimental data in maize using cross validation and validation with independent samples. Genetics, 154: 1839-1849.

Veeresha, B.A., Rudra Naik, V., Chetti, M.B., Desai, S.A. and Suma S. Biradar. 2015. QTL Mapping in Crop Plants: Principles and Applications. Int. J. Develop. Res., Vol. 5, Issue, 01, pp. 2961-2965.

Visscher, P., R. Thompson and C. Haley, 
1996. Confidence intervals in QTL mapping by bootstrapping. Genetics, 143: 1013-1020.

Weeden, N., G. Timmerman and J. Lu, 1994. Identifying and mapping genes of economic significance. Euphytica, 73: 191-198.

Weising, K., H. Nybom, K. Wolff and W. Meyer, 1995. Applications of DNA Fingerprinting in Plants and Fungi DNA Fingerprinting in Plants and Fungi, CRC Press, Boca Raton.

Winter, P. and G. Kahl. 1995. Molecular marker technologies for plant improvement. World J. Microbiol. Biotechnol., 11: 438-448.

Witcombe, J.R. and D.S. Virk, 2001. Number of crosses and population size for participatory and classical plant breeding. Euphytica, 122: 451-462.

Würschum, T., Liu, W., Gowda, M., et al. 2012. Comparison of biometrical models for joint linkage association mapping. Hered (Edinb), 108: 332-340. doi:10.1038/hdy.2011.78.

Xu, S. 1998. Mapping quantitative trait loci using multiple families of line crosses. Genetics, 148: 517-524.

Yanjun Kou and Shiping Wang. 2010. Broadspectrum and durability: understanding of quantitative disease resistance. Curr.
Opinion in Plant Biol., 13: 181-185.

Yao, H., Q. Zhou, J. Li, H. Smith, M. Yandeau, B.J. Nikolau and P.S. Schnable, 2002. Molecular characterization of meiotic recombination across the $140-\mathrm{kb}$ multigenic al-sh2 interval of nmaize. Proc. Natl. Acad. Sci. USA, 99: 61576162.

Young, N.D. 1994. Constructing a plant genetic linkage map with DNA markers, p. 39-57, In: I. K.V. Ronald and L. Phillips (Eds.), DNA-based markers in plants. Kluwer, Dordrecht/ Boston/London.

Young, N.D. 1996. QTL mapping and quantitative disease resistance in plants. Annu. Rev. Phytopathol., 34: 479-501.

Yu, J., Holland, J.B., McMullen, M.D. Buckler, E.S. 2008. Genetic design and statistical power of nested association mapping in maize. Genetics, 178: 539551. doi:10.1534/Genetics, .107 .074245 .

Zeng, Z.B. 1993. Theoretical basis for separation of multiple linked gene effects in mapping quantitative trait loci. Proc. Natl. Acad. Sci. USA, 90: 10972-10976.

Zeng, Z.B. 1994. Precision mapping of quantitative trait loci. Genetics, 136: $1457-1468$.

\section{How to cite this article:}

Fayaz Ahmad Sheikh. 2017. Recent Advances in QTL Mapping and Quantitative Disease Resistance Approach. Int.J.Curr.Microbiol.App.Sci. 6(4): 1967-1984. doi: https://doi.org/10.20546/ijcmas.2017.604.235 